\title{
Folic acid reverses uric acid crystal-induced surface OAT1 internalization by inhibiting RhoA activity in uric acid nephropathy
}

\author{
XINLIN WU ${ }^{1 *}$, JIANXIANG LIU ${ }^{2 *}$, JIANQING ZHANG ${ }^{1}$, HENG LIU $^{1}$, MIANSHENG YAN ${ }^{1}$, \\ BIRONG LIANG ${ }^{1}$, HONGBO XIE ${ }^{1}$, SHIJUN ZHANG ${ }^{1}$, BAOGUO SUN ${ }^{1}$ and HOUMING ZHOU ${ }^{1}$ \\ ${ }^{1}$ Department of Traditional Chinese Medicine, The First Affiliated Hospital, Sun Yat-sen University, \\ Guangzhou, Guangdong 510080; ${ }^{2}$ Key Laboratory of Medicinal Biotechnology, \\ Guilin Medical University, Guilin, Guangxi 541004, P.R. China
}

Received February 11, 2015; Accepted December 2, 2015

DOI: $10.3892 / \mathrm{mmr} .2016 .4837$

\begin{abstract}
To investigate how organic anion transporter (OAT)-1 is involved in uric acid nephropathy (UAN), a rat model for UAN was established and the serum uric acid, blood urea nitrogen and serum creatinine levels were all measured, and observed to be increased. It was additionally identified that in UAN rats the surface OAT1 expression levels were reduced. By treating HEK cells with monosodium urate (MSU) crystals, it was observed that the cells exhibited a reduction in OAT1 levels. Furthermore, MSU crystals were observed to recruit Ras homolog family member A (RhoA), a small guanosine triphosphatase, to the membrane and activate it. Following RhoA activation, the OAT1 internalization rate was identified to be increased. The dominant-negative RhoA N19 mutation was able to block MSU-induced OAT1 internalization, indicating that the process was RhoA-dependent. Finally, the results indicated that folic acid, a daily nutritional supplement, was capable of rescuing MSU-induced nephropathy and OAT1 internalization. These observations indicated that uric acid crystals were able to reduce the OAT1 membrane distribution through activating RhoA, and that folic acid was capable of preventing MSU-induced OAT1 relocation by inhibiting the RhoA signaling pathway.
\end{abstract}

Correspondence to: Professor Xinlin Wu, Department of Traditional Chinese Medicine, The First Affiliated Hospital, Sun Yat-sen University, 58 Second Zhongshan Road, Guangzhou, Guangdong 510080, P.R. China

E-mail:wuxinlin670522@gmail.com

*Contributed equally

Key words: uric acid crystals, OAT1, RhoA, folic acid

\section{Introduction}

Hyperuricemia is one of the most common and extensive metabolic diseases worldwide, characterized by high serum uric acid levels, resulting in the accumulation of urate crystals in the joints and kidneys $(1,2)$. Hyperuricemia is also well known as a severe risk factor for gouty arthritis, uric acid nephrolithiasis and cardiovascular and renal diseases, particularly hypertension (3-5). In humans, the level of serum uric acid is determined primarily by the ratio of urate production as an end product of the purine metabolism in liver (for which the liver enzyme xanthine oxidase is necessary) vs. elimination from the biliary and urinary tracts (6). In the urate elimination process, the organic anion transporter (OAT)-1 is hypothesized to be an essential molecule that mediates the release of urate from the blood to the basolateral membrane of renal proximal tubule (7).

The OAT family consists of 10 transmembrane proteins that are part of the solute carrier subfamily (8). Although previous studies have focused on the kidney, the OATs are localized in the barrier epithelia of the entire body, endothelium and other cells $(9,10)$. Their function is to regulate transcellular movement of numerous anionic organic molecules across the epithelium and between body fluid compartments. OAT1 is a secondary active transporter, whereby OAT-mediated influx involves exchange/counter transport of another solute involving a different transporter, such as the $\mathrm{Na}^{+}-\mathrm{K}^{+}$-ATPase $(8,9)$. OAT1 is a $p$-aminohippuric acid transporter that is responsible for the transport of several anionic drugs, toxins, metabolites and signaling molecules (11-15). Along with OAT3, OAT1 mediates the uptake of a wide range of relatively small and hydrophilic organic anions from the plasma into the cytoplasm of the proximal tubular cells of the kidneys $(15,16)$. OAT-mediated transport may also lead to renal injury by virtue of their location near the proximal tubule (17). From there, these substrates are transported into the lumen of the nephrons of the kidneys for excretion. Thus, it is suggested that OAT1 serves an important role in the regulation of urate metabolism homeostasis.

As previously reported, it has been established that a high purine diet induced a uric acid nephropathy (UAN) rat model 
that exhibited an elevated serum uric acid level in addition to kidney damage (18). Considering hyperuricemia is a key cause of UAN, and OAT1 regulates the urate metabolism and is involved in the subsequent hyperuricemia, the current study aimed to investigate whether OAT1 is involved in high purine diet-induced hyperuricemia and nephropathy in addition to the potential mechanisms.

Folic acid, which is an oxidized form of folate, is highly stable and has been used widely as a nutritional supplement. Folate deficiency is also associated with several pathologies, including neural tube defects, atherosclerosis and various types of cancer $(19,20)$. It has been demonstrated that folic acid inhibits endothelial cell proliferation through activation of the cSrc/extracellular signal-related kinase-2/nuclear factor- $\mathrm{\kappa} \mathrm{B} / \mathrm{p} 53$ pathway, which is mediated by the folic acid receptor (21). Hou et al demonstrated that folic acid-induced cSrc activation inhibited Ras homolog family member A (RhoA) activity via the activation of p190RhoGAP (22). The results demonstrated that folic acid could relocate OAT1 back to the cell membrane by inhibiting RhoA activity.

\section{Materials and methods}

Animals. A total of 40 male Sprague-Dawley rats $(250.2 \pm 1.9 \mathrm{~g}$; 6 weeks-old) were obtained from the Experimental Animal Center of the Sun Yat-sen University (Guangzhou, China). All rats were housed in plastic cages at $25^{\circ} \mathrm{C}$ under a 12 -h light-dark cycle and were provided with rodent chow and water. They were housed for one week to adapt to their environment prior to the start of the study. The study was conducted in accordance with the Guidelines for Human Treatment of Animals set by the Association of Laboratory Animal Sciences and the Center for Laboratory Animal Sciences at the Sun Yat-sen University. The current study was approved by the Committee of Biomedical Ethics of the Sun Yat-sen University [IACUC-2013-0604].

Reagents and plasmids. Allopurinol tablets were purchased from Guangzhou Kanghe Pharmaceutical Co., Ltd. (Guangzhou, China), diluted with distilled water to a final concentration of $5 \mathrm{mg} / \mathrm{ml}$. Adenine tablets were purchased from Amresco LLC (Solon, OH, USA) and diluted with $0.15 \%$ sodium carboxymethylcellulose (CMC-Na) to a final concentration of 3\%. Hematoxylin (DF001) was purchased from Guangzhou Dingguo Bio-technology Co., Ltd. (Guangzhou, China). TRIzol, reverse transcription buffer, SYBR green I polymerase chain reaction (PCR) buffer, dNTPs, MMLV and Taq DNA polymerase were purchased from Takara Biotechnology, Co., Ltd. (Dalian, China). Rabbit polyclonal OAT1 antibody purchased from Santa Cruz Biotechnology, Inc. (Dallas, TX, USA). OAT1 constructs were purchased from OriGene Technologies, Inc. (Beijing, China) in wild-type form and inserted a myc tail in the $\mathrm{C}$ terminal by routine sub-cloning (23). The RhoA construct was also purchased from OriGene Technologies, Inc. and the constitutively active RhoA G14V, and dominant-negative RhoA T19N site mutations were produced using the QuikChange Site-Directed Mutagenesis kit (with PfuUltra High-Fidelity DNA Polymerase; Agilent Technologies, Inc., Santa Clara, CA, USA) and followed by $D p n I$ (Invitrogen; Thermo Fisher Scientific, Inc., Waltham, MA, USA) digestion of the template DNA. The surface protein extraction kit was purchased from BioVersion Life Sciences (Dehradun, India), sulfo-NHS-SS-biotin and Avidin Agarose beads were purchased from Thermo Fisher Scientific, Inc. MSU crystals were produced as previously described (24).

Animal model and experimental procedures. The 40 Sprague-Dawley rats were randomly assigned into 4 groups. All rats were treated by intra-gastric administration. Group 1 was allocated as the control and was fed $5 \mathrm{ml} \mathrm{CMC-Na}$ (adenine dissolving buffer), while in the remaining 3 groups, rats were fed with adenine ( $30 \mathrm{mg} / \mathrm{kg}$ body weight) for 18 days to establish the UAN model. On the 19th day, 3 rats were randomly selected, anesthetized using $10 \%$ chloral hydrate ( $0.4 \mathrm{ml} / 100 \mathrm{~g}$; Sigma-Aldrich, St. Louis, MO, USA), sacrificed using cervical dislocation and kidney samples were collected. Hematoxylin-eosin (H\&E) staining of the kidney tissue was then conducted to assess whether the UAN model was successfully established. The 4 groups of rats were treated as follows: Group 1, blank control, rats were fed with the same amount of saline buffer; group 2, disease model control, rats were administered with the same amount of saline buffer as in group 1; group 3, allopurinol group, rats were treated with allopurinol ( $5 \mathrm{mg} / \mathrm{kg}$ body weight/day); group 4 , folic acid group, rats were treated with folic acid $(2 \mathrm{mg} / 250 \mathrm{~g}$ body weight/day). In all groups, rats were administered with the drug or saline treatment for 23 days. During the process, no rats died. Samples were collected 18 days subsequent to model establishment, and $5 \mathrm{ml}$ peripheral blood (fasting for $12 \mathrm{~h}$ ) was collected for detection of blood urea nitrogen (BUN), serum creatinine (Scr) and serum uric acid (UA). At the end of the 42-day experimental procedure, rats were fasted for $12 \mathrm{~h}$, and then sacrificed under $20 \%$ urethane anesthesia (Sigma-Aldrich). Peripheral blood was collected to detect BUN, Scr and UA, and the left kidney was resected for further investigation.

$H \& E$ staining. Kidney sections were stained with hematoxylin for $2 \mathrm{~min}$ and then eosin for $30 \mathrm{sec}$. Subsequent to washing, the slides were dehydrated and sealed with Entellan (Merck Millipore, Darmstadt, Germany) prior to examination under a light microscope (model BX43/53; Olympus Corporation, Tokyo, Japan). Quantification of cell densities was conducted using AlphaEaseFC software version 4.0 (Alpha Innotech Corporation, San Leandro, CA, USA). Results were analyzed using Image Pro Plus software, version 6.0 (Media Cybernetics, Inc., Rockville, MD, USA), and in each section 10 fields were selected (magnification, x200).

Tissue surface protein extraction, cell surface biotinylation and western blotting. Kidney tissues from rats in the different groups were frozen and stored at $-20^{\circ} \mathrm{C}$ immediately subsequent to dissection. The tissues were mechanically dissociated and homogenized (model AH96; Omni International, Kennesaw, GA, USA) following the manufacturer's instructions to obtain the surface protein. For HEK cell (American Type Culture Collection, Manassas, VA, USA) surface biotinylation, OAT1-stable HEK cells were treated with monosodium urate (MSU) for $24 \mathrm{~h}$ and washed twice with cold phosphate-buffered saline (PBS). OAT1-stable HEK cells without MSU treatment were used as a control. The 
cells were then incubated with PBS supplemented with $0.5 \mathrm{mg} / \mathrm{ml}$ sulfo-NHS-SS-biotin for $1 \mathrm{~h}$ with gentle shaking, and excess biotin was quenched with $50 \mathrm{mM}$ Tris-PBS buffer (Sigma-Aldrich) for $20 \mathrm{~min}$. Cells were lysed in radioimmunoprecipitation assay (RIPA) buffer and then were subjected to streptavidin-agarose beads at $4^{\circ} \mathrm{C}$ for an additional $3 \mathrm{~h}$. For the HEK cell internalization assay, surface biotinylated cells were incubated at $37^{\circ} \mathrm{C}$ for $30 \mathrm{~min}$, subsequent to quenching, to allow internalization, then were washed with cold cleavage buffer [50 mM glutathione, $90 \mathrm{mM} \mathrm{NaCl}, 1.25 \mathrm{mM} \mathrm{CaCl}_{2}$ dihydrate, $1.25 \mathrm{mM} \mathrm{MgSO}_{4}$ (all from Sigma-Aldrich), and $0.2 \%$ endotoxin-free bovine serum albumin (BSA; Cell Signaling Technology, Inc., Danvers, MA, USA), pH 8.6] for 20 min with gentle shaking. Cells were lysed in RIPA buffer, then were subjected to streptavidin-agarose beads at $4^{\circ} \mathrm{C}$ for another $3 \mathrm{~h}$. Protein samples were boiled in Laemmli buffer (Amresco LLC), separated by $12 \%$ sodium dodecyl sulphate-polyacrylamide gel electrophoresis (ProSpec, Ness-Ziona, Israel), then transferred to polyvinylidene difluoride membranes (Hybond-C-extra; GE Healthcare Life Sciences, Chalfont, UK). Membranes were blocked using 5\% BSA in Tris-buffered saline with Tween-20 (TBST; Sigma-Aldrich) and then were incubated overnight at $4^{\circ} \mathrm{C}$ with mouse monoclonal anti-OAT1 (1:100; cat. no. ab118346; Abcam, Cambridge, MA, USA) or rabbit monoclonal anti-glyceraldehyde 3-phosphate dehydrogenase (GAPDH) (1:8,000; cat. no. ab181603; Abcam) antibodies. The membranes were then washed in TBST and incubated with peroxidase-conjugated goat anti-rabbit or anti-mouse antibodies (1:4,000; Jackson ImmunoResearch Laboratories, Inc., West Grove, PA, USA) for $1 \mathrm{~h}$. Protein bands were developed using Enhanced Chemiluminescence plus reagent (Western Lightning; PerkinElmer, Inc., Waltham, MA, USA).

Detection of OATI mRNA expression by reverse transcription-quantitative $P C R(R T-q P C R)$. Total RNA $(2 \mu \mathrm{g})$ was extracted with TRIzol (Invitrogen; Thermo Fisher Scientific, Inc.) and converted to cDNA $(1 \mu \mathrm{g})$ using the reverse transcription kit (Takara Biotechnology, Co., Ltd.) according to the manufacturer's instructions. RT-qPCR was conducted according to the following procedure: $94^{\circ} \mathrm{C}$ for $3 \mathrm{~min}$, followed by 40 cycles of $94^{\circ} \mathrm{C}$ for $30 \mathrm{sec}, 55^{\circ} \mathrm{C}$ for $45 \mathrm{sec}$ and $72^{\circ} \mathrm{C}$ for 1 min. OAT1, F 5'-CAGCCAAGGAGGCTGCTGTC-3' and R 5'-AGTCAAACCTTTTAATGATG-3'; GAPDH, F 5'-TGG TCTACATGTTCCAGTATGACT-3' and R 5'-CCATTTGAT GTTAGCGGGATCTC-3'. Data were presented as the normalized OAT1 mRNA level/GAPDH mRNA level. Results are expressed as the mean \pm standard error of three independent experiments.

Surface and internalization OAT1 staining. OAT1 stable HEK cells were treated with or without MSU for $24 \mathrm{~h}$ and live cells were used for staining the OAT1 extracellular region at $4^{\circ} \mathrm{C}$ for $1 \mathrm{~h}$. Cells were then transferred back to the $37^{\circ} \mathrm{C}$ incubator for $30 \mathrm{~min}$ to allow for internalization. Cells were fixed with 4\% paraformaldehyde (Sigma-Aldrich) and surface stained using the Alexa Fluor 488 donkey anti-goat polyclonal antibody (1:200; cat. no. ab150129; Abcam) for $1 \mathrm{~h}$. Subsequently, the cells were permeabilized with $0.25 \%$ Triton X-100 (Sigma-Aldrich) for $5 \mathrm{~min}$ and incubated with the mouse monoclonal anti-Myc antibody (1:1,000; cat. no. ab23; Abcam) at $4^{\circ} \mathrm{C}$ overnight. On the second day, cells were stained with the Alexa Fluor 594 donkey anti-goat antibody (1:300; cat. no. ab150132; Abcam) for internalized OAT1 and Alexa Fluor 405 goat anti-mouse polyclonal antibody (1:200; cat. no. ab175660; Abcam) for total OAT1. Representative images were captured using an Olympus FV1000 confocal microscope (Olympus Corporation, Tokyo, Japan).

Statistical analysis. Quantitative data was expressed as the mean \pm standard error. Comparisons of the means among multiple groups were performed using a one-way analysis of variance followed by Dunnett's or Tukey-Kramer's post hoc tests using GraphPad Prism software, version 4.0 (GraphPad Software, Inc., La Jolla, CA, USA). Quantification of images were conducted using Image J software (National Institutes of Health, Bethesda, MD, USA). Asterisks were used to indicate the following: ${ }^{*} \mathrm{P}<0.05,{ }^{* *} \mathrm{P}<0.01$ and ${ }^{* * *} \mathrm{P}<0.001$. $\mathrm{P}<0.05$ was considered to indicate a statistically significant difference.

\section{Results}

UAN model rats exhibited a reduction in surface OATI expression. Compared with the controls, serum UA, BUN and creatinine levels in adenine-treated rats (UAN model rats) were significantly increased, suggesting that the UAN model had been successfully established. Treatment with allopurinol led to a reduction in serum UA, BUN and creatinine levels compared with the UAN model group, however, serum BUN and creatinine levels of allopurinol-treated rats remained significantly greater than those of the control group (Fig. 1A-C). Using H\&E staining, it was additionally identified that UAN model rats exhibited kidney damage (14) (renal interstitial tissue was characterized by edema, inflammatory cell infiltration, granuloma hyperplasia, glomeruli exhibiting partial atrophy and tubular cells with diffuse swelling), while treatment with allopurinol attenuated these kidney impairments (Fig. 1D). The above-mentioned data indicate that the model was successful as previously reported (18).

To further investigate the role of OAT1 in UAN, the OAT1 protein expression levels were measured. As presented in Fig. 1E, no significant different in the total OAT1 level was observed between the different groups, and the mRNA levels of OAT1 were not altered (Fig. 1F). As OAT1 is a membrane protein, the total plasma membrane proteins were extracted, and the surface OAT1 levels were measured. It was identified that although total OAT1 expression was not altered, there was a significant reduction in the level of surface OAT1 in the UAN group (Fig. 1G). The results indicated that high uric acid generation may reduce the surface OAT1 levels.

Uric acid crystals reduce surface levels of OAT1 by facilitating its internalization. To further investigate the detailed mechanism of how uric acid regulates OAT1 expression or trafficking, OAT1 stable expressing HEK cells were used and they were treated with or without MSU crystals $(1 \mu \mathrm{M})$ for $24 \mathrm{~h}$. The results demonstrated that treatment with MSU crystals resulted in a reduction in surface OAT1 levels however not in the total protein (Fig. 2A) or mRNA levels (Fig. 2B). The internalized OAT1 levels were then measured, and it was 
A

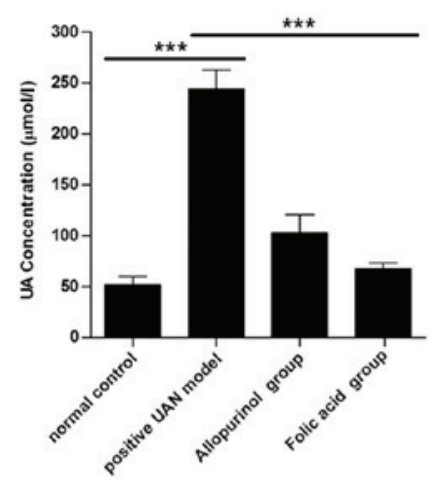

D
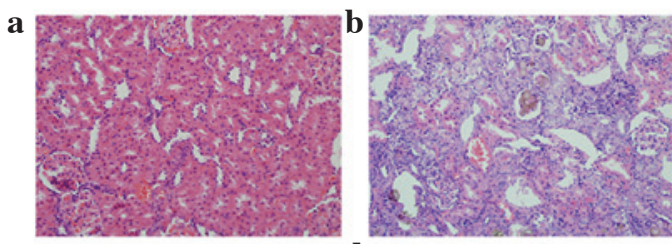

$\mathbf{c}$
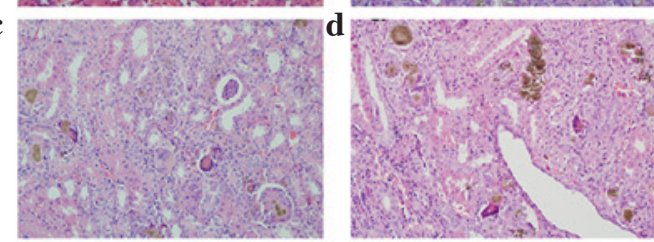

F

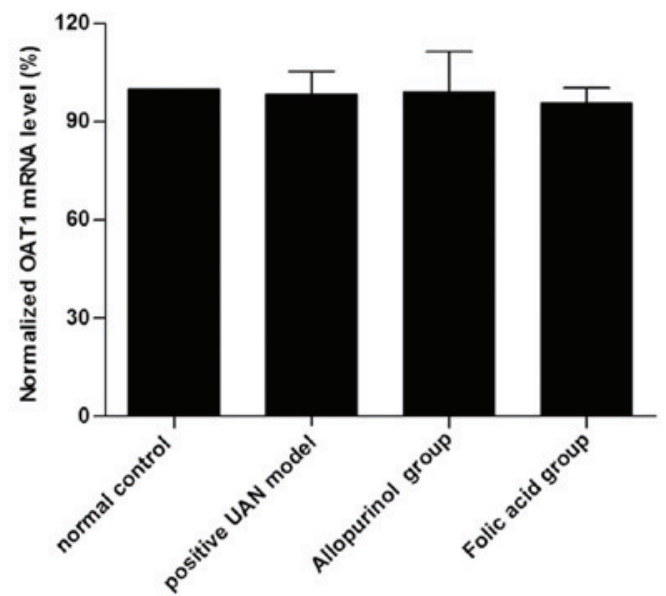

B

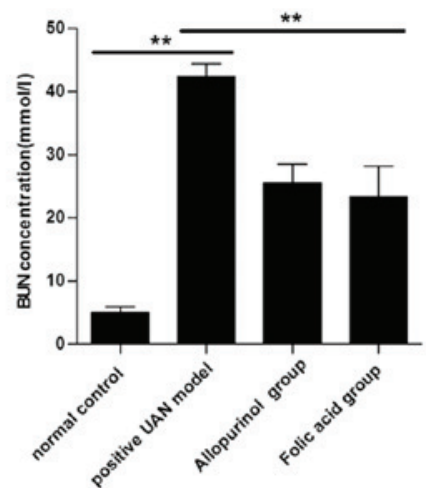

C

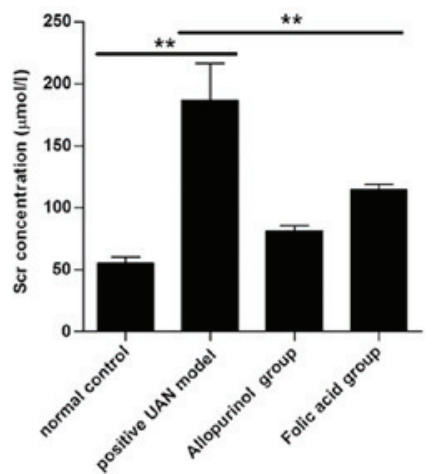

E

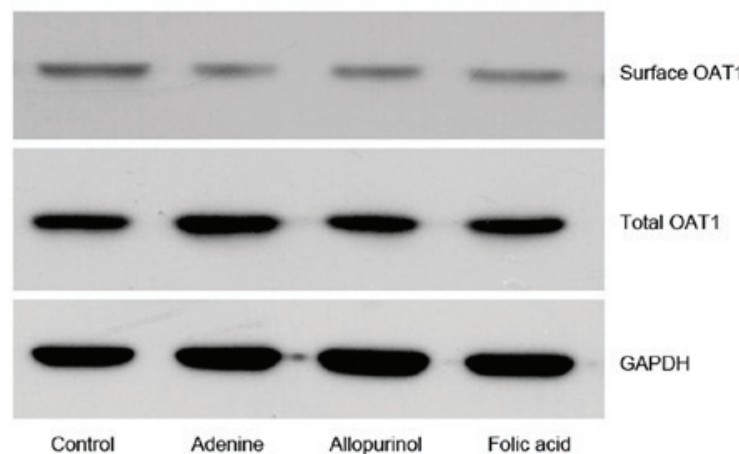

G

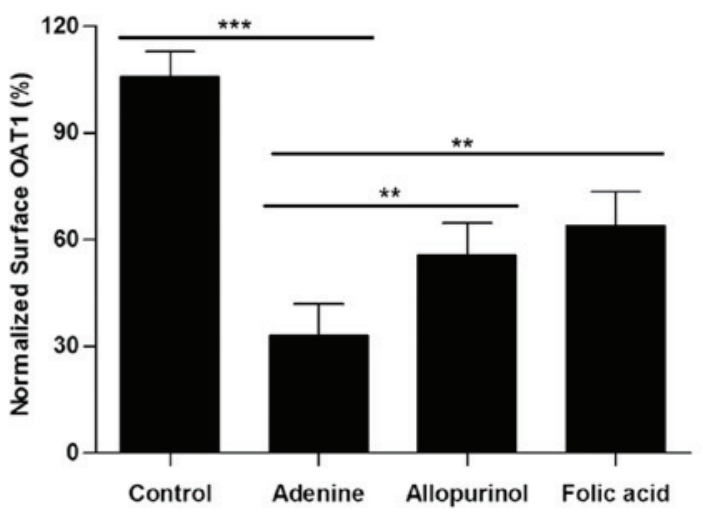

Figure 1. UAN model rats exhibited a reduction in surface OAT1 levels in kidney tissues. Histograms for the serum (A) UA, (B) BUN, and (C) Scr levels in the UAN rats are presented. (D) Light microscopy images of kidney tissue sections with H\&E staining (magnification, x200): (a) Control, (b) UAN model, (c) allopurinol and (d) folic acid. (E) Representative western blot of surface OAT1 and total OAT1 from rat kidney tissues. GAPDH was used as a loading control. (F) Reverse transcription-quantitative polymerase chain reaction results of OAT-1 mRNA expression levels from various groups. (G) Histogram of quantification of OAT1 expression levels, normalized to GAPDH. Data represent the mean \pm standard error $\left({ }^{* *} \mathrm{P}<0.01,{ }^{* * * *} \mathrm{P}<0.001\right)$. UAN, uric acid nephropathy; OAT1, organic anion transporter-1; UA, serum uric acid; BUN, blood urea nitrogen; Scr, serum creatinine; GAPDH, glyceraldehyde 3-phosphate dehydrogenase.

identified that following treatment with MSU crystals, OAT1 internalization was increased (Fig. 2C and D). Notably, it was observed that treatment of HEK cells exposed to MSU with folic acid led to a reversal of these effects (Fig. 2A-D). Using confocal imaging it was also confirmed that although total OAT1 was not significantly altered (Fig. 2E), the reduction in surface expression levels corresponded with an increase in OAT1 internalization, while treatment with folic acid reversed this effect (Fig. 2F).

Uric acid crystals activate RhoA and induce OAT1 internalization. How the OAT1 trafficking process is regulated was then investigated. Considering that the cytoskeleton is a major component that controls vesicular movement, the experiments focused upon the small guanosine triphosphatase, RhoA, which is known to regulate actin-stress fiber formation and facilitates endocytosis following activation $(24,25)$. HEK cells were treated with uric acid crystals, and it was identified that although total RhoA levels were not significantly altered, the guanosine triphosphate bound form of RhoA (active form) was increased significantly (Fig. 3A B, C and D). Notably, treatment with folic acid led to a reversal in RhoA levels. To further investigate the effects of RhoA on OAT1 internalization, constitutively active 


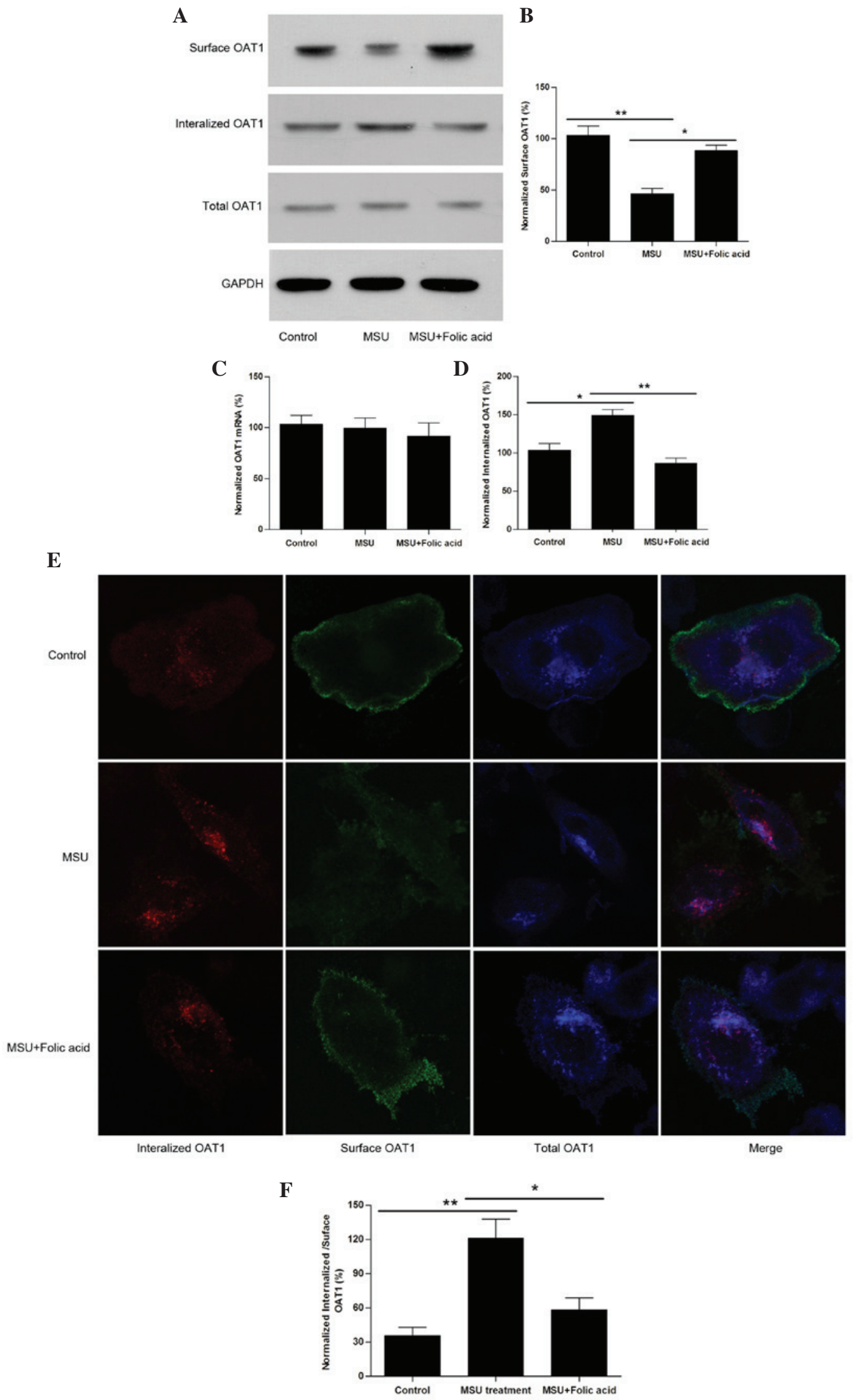

Figure 2. Uric acid crystal alter OAT1 trafficking in HEK cells. (A) Representative western blot of surface, internalized and total OAT1 levels by biotin assay. (B) Reverse transcription-quantitative polymerase chain reaction results for OAT1 mRNA expression levels with or without MSU crystals or folic acid treatment. (C) Histograms demonstrating the quantification of surface or internalized OAT1 expression levels normalized with GAPDH. (D) Histogram showing quantification of internalized OAT1 expression levels, normalized to GAPDH. (E) Confocal microscopy images of live cell staining for surface (green), internalized (red) or total OAT1 (blue) levels. (F) Histogram showing quantification of the ratio of surface to internalized OAT1, normalized with total OAT1 levels. Data are presented as the mean \pm standard error ( $\mathrm{P}<0.05$, "** $\mathrm{P}<0.01)$. OAT1, organic anion transporter-1; MSU, monosodium urate; GAPDH, glyceraldehyde 3-phosphate dehydrogenase. 
A

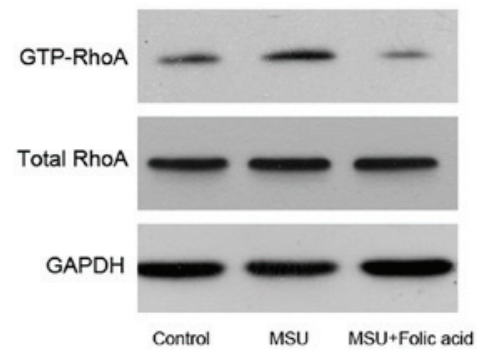

B

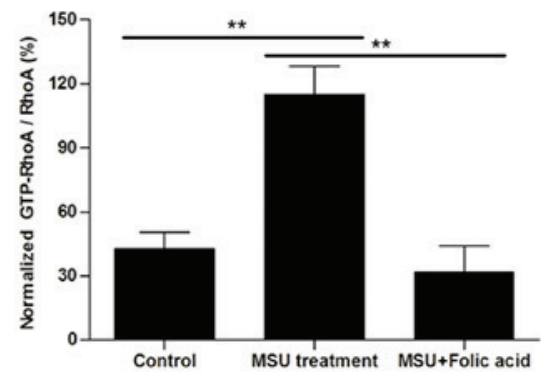

MSU
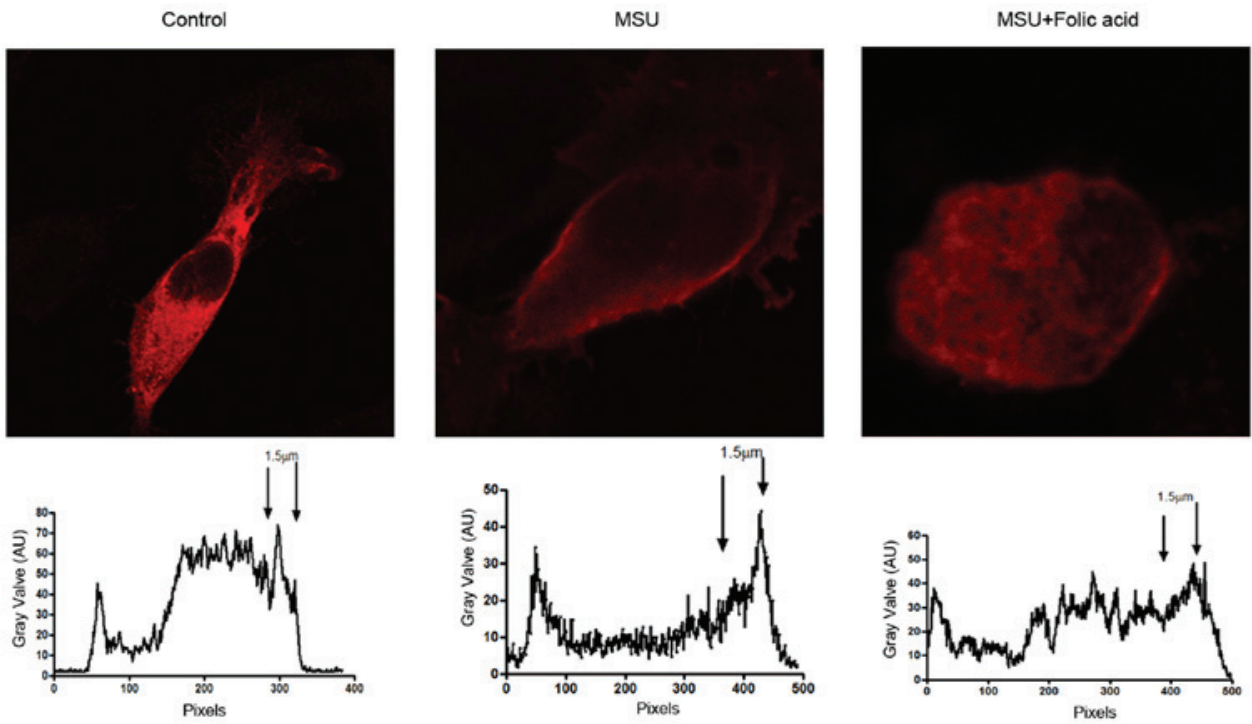

D

E

GTP-RhoA
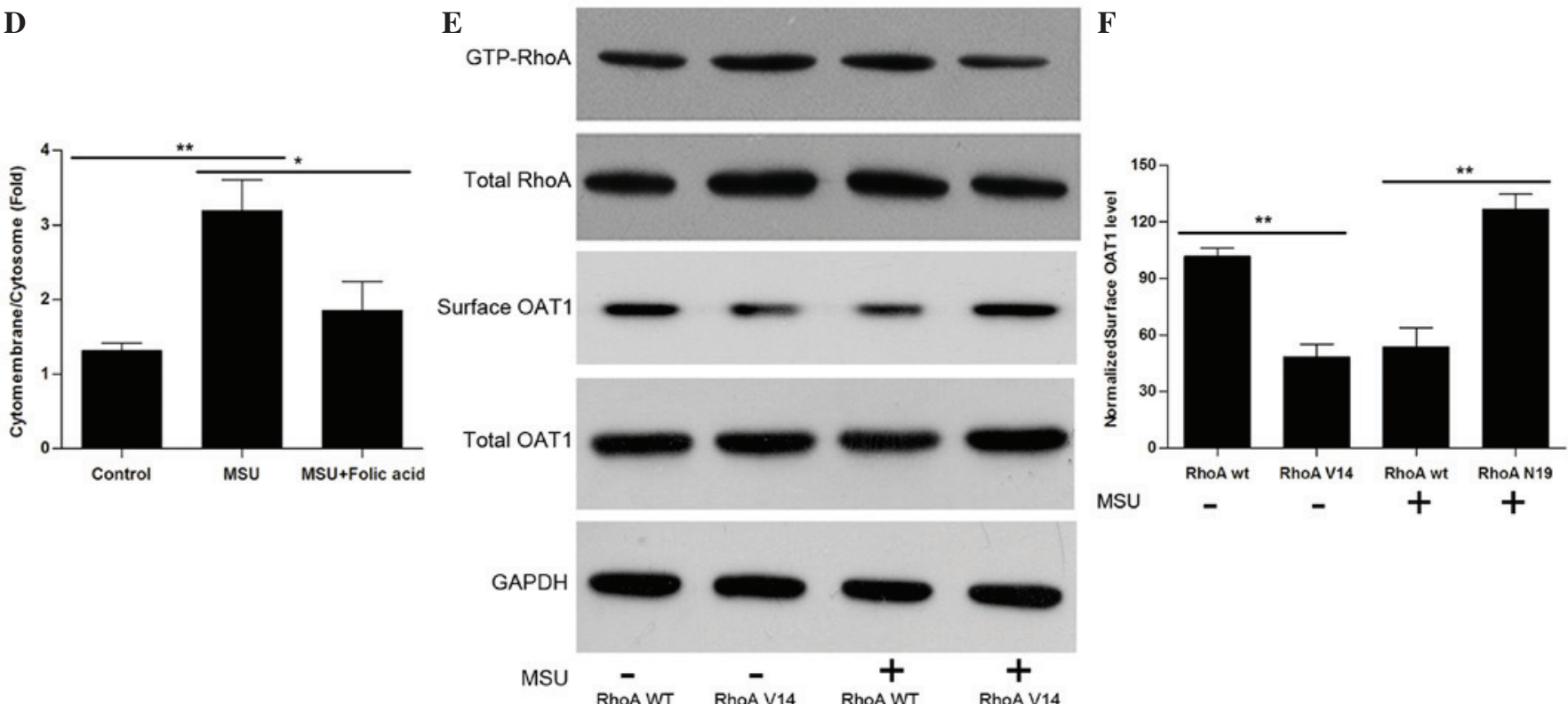

$\mathbf{F}$

Figure 3. Uric acid crystals regulate OAT1 trafficking through the activation of RhoA. (A) Western blot analysis of active and total RhoA in OAT1 stable HEK cells with or without MSU and folic acid treatment. (B) Quantification of active RhoA expression levels, normalized to total RhoA. (C) Confocal images of plot profile analysis for RhoA distribution in OAT1 stable HEK cells with or without MSU and folic acid treatment. (D) Quantification of cytomembrance RhoA expression levels, normalized to cytosome RhoA. (E) Western blot analysis of GTP-RhoA and total RhoA, surface and total OAT1 expression in RhoA V14 and N19 mutation co-treated with or without MSU. (F) Quantification of the ratio of surface and internalized OAT1, normalized to GAPDH. Data are presented as the mean \pm standard error ( $\left.\mathrm{P}<0.05,{ }^{* *} \mathrm{P}<0.01\right)$. OAT1, organic anion transporter-1; RhoA, Ras homolog gene family member A; MSU, monosodium urate; GTP, guanosine triphosphate; GAPDH, glyceraldehyde 3-phosphate dehydrogenase; WT, wild-type.

RhoA G14V and dominant-negative RhoA T19N site mutation constructs were produced. By overexpression of these constructs in OAT1-HEK cells, RhoA V14 was identified to lead to an increase in OAT1 internalization without crystal stimulation, while RhoA N19 inhibited uric acid-induced OAT1 internalization (Fig. 3E and F). 
Folic acid was able to rescue OAT1 internalization by inhibiting RhoA activity. As it has been previously reported that folic acid was able to inhibit RhoA activity in cell models (13), the current study aimed to demonstrate whether folic acid possesses benefits on high uric acid-induced kidney damage. The results demonstrated that daily supplements of folic acid were able to rescue uric acid induced kidney damage and repress serum uric acid, BUN and creatinine levels by restoring the levels of surface OAT1 back to normal levels in vivo (Fig. 1). Treatment with MSU-treated HEK cells with or without folic acid $(10 \mu \mathrm{M})$ demonstrated that folic acid was able to rescue OAT1 membrane distribution by inhibiting RhoA activation (Figs. 2 and 3).

\section{Discussion}

In the present study, it was suggested that urate transporter OAT1 may be involved in high purine-induced kidney damage in rats. The results of the current study suggested that a purine rich diet may lead to kidney damage through high serum uric acid, and that serum uric acid could markedly reduce surface OAT1 expression levels. In addition, folic acid, which could restore OAT1 surface expression back to normal, was able to rescue uric acid-induced kidney damage. In HEK cells, it was identified that high concentrations of uric acid were able to reduce cell surface OAT1 expression levels, however not the total OAT1 protein amount nor its mRNA level. As OAT1 serves an important role in uric acid release, the results here indicated that the reduction in surface OAT1 may be involved in high serum uric acid-induced kidney impairments as a second step following serum uric acid hyper-production. This in turn may lead to aggravation of the urate metabolism dyshomeostasis and serum uric acid accumulation. Furthermore, it was identified that uric acid stimulation may facilitate OAT1 internalization through RhoA activation, and that folic acid was able to rescue surface OAT1 distribution by inhibiting RhoA activation.

Rho proteins, including Cdc42, Rac1 and RhoA, have been best characterized for their effects on the cytoskeleton and cell adhesion. Subsequent to activation, RhoA would be recruited from the cytosome to the membrane region, and then activate downstream signals through phosphorylation. Activation of RhoA is recognized to mediate the formation of stress fibers and focal contacts that firmly anchor cells to their substrata, permitting retraction of cellular rear ends by mediating integrin endocytosis $(20,21)$. In addition to integrin, active RhoA was able to mediate the endocytosis of numerous other proteins, such as Toll-like receptor-4, $\beta$-arrestin-1 or the Kv1.2 potassium channel (16-18). The mechanism may involve regulating actin dynamic polymerization and selected docking of transport vesicles. Although no clear reports have elucidated how uric acid may activate RhoA, a previous study demonstrated that MSU treatment could relocate RhoA to the membrane region (19), which indicated that uric acid may activate RhoA through currently unidentified pathways.

Folic acid, an important daily nutritional supplement which has functions in pregnancy, cancer, stroke or heart disease, has been identified to suppress RhoA activation through the folic acid receptor/cSrc/p190RhoGAP-signaling pathway (15).
The current study identified that folic acid was also able to inhibit uric acid-induced RhoA activation, and that this may regulate RhoA activity through the same pathway by altering the phosphorylation status of the RhoA upstream regulator, p190RhoGAP.

In summary, the present study demonstrated, for the first time to the best of our knowledge, that in UAN, uric acid stimulation can reduce OAT1 membrane expression by facilitating its endocytosis, which was regulated by activated RhoA, and this could be rescued by folic acid treatment. A potential mechanism by which high concentration of serum uric acid would induce UAN was described, which suggests that folic acid may have potential therapeutic value.

\section{Acknowledgements}

The current study was supported by the Natural Science Foundation of Guangdong Province (grant no.2011B080701007).

\section{References}

1. Klemp P, Stansfield SA, Castle B and Robertson MC: Gout is on the increase in New Zealand. Ann Rheum Dis 56: 22-26, 1997.

2. Arromdee E, Michet CJ, Crowson CS, O'Fallon WM and Gabriel SE: Epidemiology of gout: Is the incidence rising? J Rheumatol 29: 2403-2406, 2002.

3. Li Y, Stamler J, Xiao Z, Folsom A, Tao S and Zhang H: Serum uric acid and its correlates in Chinese adult populations, urban and rural, of Beijing. The PRC-USA collaborative study in cardiovascular and cardiopulmonary epidemiology. Int J Epidemiol 26: 288-296, 1997.

4. Johnson RJ, Kang DH, Feig D, Kivlighn S, Kanellis J, Watanabe S, Tuttle KR, Rodriguez-Iturbe B, Herrera-Acosta J and Mazzali M: Is there a pathogenetic role for uric acid in hypertension and cardiovascular and renal disease? Hypertension 41: 1183-1190, 2003.

5. Tseng CH: Independent association of uric acid levels with peripheral arterial disease in Taiwanese patients with Type 2 diabetes. Diabet Med 21: 724-729, 2004.

6. Kai H, Kaneyuki M, Shihara M, Toyama Y, Mitsutake Y, Umei H, Kusaba K, Ueda T, Adachi H and Imaizumi T; MAPPY Study Investigators: Reduction in morning blood pressure is a key factor for ameliorating urinary albumin excretion in patients with morning hypertension irrespective of treatment regimen. Circ J 77: 1551-1557, 2013.

7. Sekine T, Cha SH and Endou H: The multispecific organic anion transporter (OAT) family. Pflugers Arch 440: 337-350, 2000.

8. Koepsell H: The SLC22 family with transporters of organic cations, anions and zwitterions. Mol Aspects Med 34: 413-435, 2013.

9. Sweeney DE, Vallon V, Rieg T, Wu W, Gallegos TF and Nigam SK: Functional maturation of drug transporters in the developing, neonatal, and postnatal kidney. Mol Pharmacol 80: 147-154, 2011.

10. Ulmius M, Johansson-Persson A, Krogh M, Olsson P and Önning G: An oat bran meal influences blood insulin levels and related gene sets in peripheral blood mononuclear cells of healthy subjects. Genes Nutr 6: 429-439, 2011.

11. Pritchard JB: Coupled transport of p-aminohippurate by rat kidney basolateral membrane vesicles. Am J Physiol 255: F597-F604, 1988.

12. Sweet DH, Wolff NA and Pritchard JB: Expression cloning and characterization of ROAT1. The basolateral organic anion transporter in rat kidney. J Biol Chem 272: 30088-30095, 1997.

13. Burckhardt G: Drug transport by organic anion transporters (OATs). Pharmacol Ther 136: 106-130, 2012.

14. Pavlova A, Sakurai H, Leclercq B, Beier DR, Yu AS and Nigam SK: Developmentally regulated expression of organic ion transporters NKT (OAT1), OCT1, NLT (OAT2), and Roct. Am J Physiol Renal Physiol 278: F635-F643, 2000.

15. VanWert AL, Gionfriddo MR and Sweet DH: Organic anion transporters: Discovery, pharmacology, regulation and roles in pathophysiology. Biopharm Drug Dispos 31: 1-71, 2010. 
16. Kim S, Lee CH, Kang CM and Kim GH: Effects of increased uric acid intake on the abundance of urate-anion exchanger and organic anion transporter proteins in the rat kidney. Electrolyte Blood Press 5: 62-67, 2007.

17. Yarlagadda SG and Perazella MA: Drug-induced crystal nephropathy: An update. Expert Opin Drug Saf 7: 147-158, 2008

18. Wu X, Liu L, Xie H, Liao J, Zhou X, Wan J, Yu K, Li J and Zhang Y: Tanshinone IIA prevents uric acid nephropathy in rats through NF-кB inhibition. Planta Med 78: 866-873, 2012.

19. Kim YI: Will mandatory folic acid fortification prevent or promote cancer? Am J Clin Nutr 80: 1123-1128, 2004.

20. Choi SW and Mason JB: Folate status: Effects on pathways of colorectal carcinogenesis. J Nutr 132 (Suppl 8): S2413-S2418, 2002.

21. Lin SY,Lee WR, Su YF,Hsu SP,Lin HC,Ho PY,Hou TC, Chou YP, Kuo CT and Lee WS: Folic acid inhibits endothelial cell proliferation through activating the cSrc/ERK $2 / \mathrm{NF}-x \mathrm{~B} / \mathrm{p} 53$ pathway mediated by folic acid receptor. Angiogenesis 15: 671-683, 2012.
22. Hou TC, Lin JJ, Wen HC, Chen LC, Hsu SP and Lee WS: Folic acid inhibits endothelial cell migration through inhibiting the RhoA activity mediated by activating the folic acid receptor/cSrc/p190RhoGAP-signaling pathway. Biochem Pharmacol 85: 376-384, 2013.

23. Fromme $\mathrm{T}$ and Klingenspor M: Rapid single step subcloning procedure by combined action of type II and type IIs endonucleases with ligase. J Biol Eng 1: 1-3, 2007.

24. Shi Y, Evans JE and Rock KL: Molecular identification of a danger signal that alerts the immune system to dying cells. Nature 425: 516-521, 2003.

25. Etienne-Manneville S and Hall A: Rho GTPases in cell biology. Nature 420: 629-635, 2002 